\section{$\$$ Research Square \\ Preprints are preliminary reports that have not undergone peer review. \\ They should not be considered conclusive, used to inform clinical practice, or referenced by the media as validated information.}

\title{
Single-nucleotide polymorphisms in one-carbon metabolism genes, Mediterranean diet and the risk of pre- and postmenopausal breast cancer
}

\section{Shang Cao}

Southeast University

Zheng Zhu

Jiangsu Province Center for Disease Control and Prevention

Jinyi Zhou

Jiangsu Province Center for Disease Control and Prevention

Wei Li

Southeast University

\section{Yunqiu Dong}

Wuxi Center for Disease Control and Prevention

Yun Qian

Wuxi Center for Disease Control and Prevention

Pingmin Wei

The University of Melbourne Asia Institute

Ming Wu ( $\nabla$ mingwu@seu.edu.cn)

Jiangsu Province Center for Disease Control and Prevention https://orcid.org/0000-0002-8798-0288

\section{Research}

Keywords: Breast cancer; Mediterranean dietary pattern (MDP), One-carbon metabolism, Single nucleotide polymorphisms (SNP)

Posted Date: June 25th, 2020

DOI: https://doi.org/10.21203/rs.3.rs-26394/v2

License: @) (1) This work is licensed under a Creative Commons Attribution 4.0 International License. Read Full License 


\section{Abstract}

Background: One-carbon metabolism pathway could interfere with the carcinogenesis of breast cancer (BC). Dietary pattern plays an important role in onecarbon metabolism linking the combination of dietary micronutrients.

Methods: We assessed associations between single-nucleotide polymorphisms (SNPs) of one-carbon metabolism, adherence to Mediterranean dietary pattern (MDP) and BC risk from a case-control study (818 cases, 935 controls) among Chinese female population. The genotyping of 10 SNPs in seven one-carbon metabolism-related genes (MTHFD1, MTRR, MAT2B, CDO1, FOLR1, UNG2, ADA) were performed. Dietary intake measurements were assessed by a validated food-frequency questionnaire. Gene-diet interactions were analyzed.

Results: No evidence demonstrated SNPs of one-carbon metabolism or their haplotypes were associated with BC risk. High adherence to the Mediterranean dietary pattern decreased the risk of breast cancer among post- but not premenopausal women and the association was influenced by the SNPs genotype, with the increasing number of variant allele in MTHFD1 (rs 11627387), MTHFD1 (rs2281603), MTRR (rs16879334), MTRR (rs2287780), MAT2B (rs4869087), FOLR1 (rs 10501409), UNG2 (rs231622) and ADA (rs244072). The protective effect against BC risk from high adherence to the MDP was gradually weakened and disappeared. For MTHFD1 (rs8003567) and CDO1 (rs34869) genotypes, women with homozygous were less affected by adherence to the MDP than to women who with heterozygotes. No significant gene-diet interactions were observed.

Conclusions: SNPs of one-carbon metabolism genes modify the effect of high adherence to MDP against BC risk in Chinese women, as potential effect modifiers. Genetic variants may influence the association between diet and BC risk.

\section{Introduction}

The primary risk factors of breast cancer $(\mathrm{BC})$ include overweight or obesity, physical inactivity, exogenous hormone intake (use of oral contraceptive and hormone replacement therapy), reproduction condition (late age at first birth and low parity), family history of cancer and unhealthy diet(Bethea, et al. 2015, Brewer, et al. 2017, Deschasaux, et al. 2017, Ellingjord-Dale, et al. 2017, Neuhouser, et al. 2015, Trinh, et al. 2015). Besides these modifiable risk factors, genetic predisposition also plays an important role in susceptibility to BC. Inherited mutations in BRCA1 and BRCA2 genes confer a high risk of $\mathrm{BC}$ to women of specific families(Torres, et al. 2017), whereas, for a large percentage of cases, BC is more likely attributed to a combination of genetic variations and environmental or lifestyle factors. Elucidating the gene-environment interactions is therefore essential to unveil the molecular mechanisms involved in breast carcinogenesis.

Diet is one of the environmental factors and often related to individuals' health outcomes. A well-balanced dietary pattern, such as the Mediterranean diet, is able to reduce the risks of a variety of diseases. The traditional Mediterranean diet is characterized by the liberal use of olive oil; the high consumption of vegetables, fruits, nuts, legumes and unprocessed cereals; the moderate amounts of fish and wine; the low intake of dairy products (with the exception of cheeses), red meat and meat products(Trichopoulou, et al. 2014). A number of epidemiological studies have suggested that this dietary pattern had substantial health benefits and displayed inverse associations with multiple disease types, including cancer(Soltani, et al. 2019). Our study also showed that high adherence to the Mediterranean dietary pattern significantly reduced post-menopausal BC risk in Chinese women. Since cancer development is generally determined by the interplay between extrinsic and intrinsic factors, the roles of underlying molecular pathways in the association of the Mediterranean diet with $\mathrm{BC}$ risk require further investigation.

One-carbon metabolism is a complicated metabolic network composed of cascade reactions based on the transfer of one-carbon units, which is required by nucleotide synthesis, DNA replication, repair and methylation. Folate, a water-soluble vitamin-B found rich in leafy green vegetables and fruits, beans and peas, grains and cereals, is an essential component of this metabolism pathway as the functional donor of one-carbon units. The inadequate intake of nutrients in the daily diet could destroy the normal physiological process of one-carbon metabolism, associated with the disruption of DNA replication and repair as well as aberrant DNA methylation patterns, eventually leading to carcinogenesis(Miranti, et al. 2017). Some epidemiological studies provide some promising results, which focused on MTHFR (rs1801131, rs1801133) and MTR (rs1805087), intake of folate and vitamin B6/B12 and BC risk(Ma, et al. 2009, Platek, et al. 2009). Also, genetic polymorphisms in the one-carbon metabolism pathway genes encoding functional enzymes and coenzymes have been suggested to influence individuals' susceptibility to cancer, such as Methylenetetrahydrofolate reductase (MTHFR) and methionine synthase (MTR) (Shao, et al. 2018, Wu, et al. 2016). However, in addition to these most common polymorphisms, the role of genetic variants in other one-carbon metabolism genes and their interactions with diet regarding BC risk remain largely unexplored, especially in the Asian female population.

In this study, we evaluated associations between genetic polymorphisms in one-carbon metabolism-related genes and the risk of BC among Chinese women. We further investigated the interaction effects of selected genetic variants with high adherence to the Mediterranean dietary pattern on BC carcinogenesis.

\section{Materials And Methods}

\section{Subjects}

Subjects were from the Chinese Wuxi Exposure and Breast Cancer Study (2013-2014), a population-based case-control study of the role of biology, diet, lifestyle, and environmental factors in the etiology of BC in Asian women. The subjects were all women who lived in Wuxi city, Jiangsu Province, China, for more than five years. Newly diagnosed breast cancer patients within one year were selected as the case group according to the local cancer registration system. All cases were identified according to the International Classification of Diseases for Oncology (ICD-10, code C50) and were confirmed by pathology, excluding patients with secondary or recurrent BC. For those with multiple incident cancers, only included those with BC as the first original malignancy diagnosed. Controls were matched to the cases in a ratio of $1: 1$ by the same residence area and age (range of \pm 5 years), excluding individuals with any cancer 
history. The study protocol was approved by the Institutional Review Boards of Jiangsu CDC, and the informed consent were obtained from all subjects. Blood samples were collected from both cases and controls.

\section{Data on diet}

The usual diet was assessed by a validated, semi-quantitative food frequency questionnaire (FFQ), which included 149 items along with the recipes commonly used in China(Zhao, et al. 2002). Nutrient and energy intake were calculated through the Chinese Food Composition Database. Dietary intake assessment included whether the food was consumed, consumption frequency (times of per day/week/month/year) and the average amount of food consumption at each time. The 149 food items in the FFQ were classified into 18 predefined food groups based on similarities in nutrient profile and culinary usage.

\section{Mediterranean diet scale}

The Alternate Mediterranean Diet Score (aMED) established by Fung et al.,(Fung, et al. 2005) includes nine dietary components and range from 0-9 scores (minimum to maximum conformity). One point is given to each subject when the food intake is equal to or above the median intake of controls for the following seven components regarded as healthy: grains, fruits, vegetables, legumes, nuts, fish, and monounsaturated fat-saturated fat ratio. One point is given when the intake amount of a subject is less than the median intake of unhealthy food such as red meat or processed meat, or alcohol consumption within 5-25 g/day for women as a specified range. The higher the score obtained from the questionnaire, the greater the adherence to the Mediterranean dietary pattern (MDP).

\section{Lifestyle, anthropometric, medical history and reproductive history data}

Demographic, lifestyle characteristics, menstrual and reproductive events, dietary intake, disease history and physical activity-related data were collected from a structured questionnaire, through in-person interviews conducted by trained interviewers. Anthropometric measures were obtained by trained personnel following the protocol. Physical activity was measured by referencing the Global Physical Activity Questionnaire. Postmenopause was defined as an absence of menstruation in the past 12 months.

\section{Genotyping assays}

A total of 15 single-nucleotide polymorphisms (SNPs) involved in 11 one-carbon metabolism genes including Methylenetetrahydrofolate dehydrogenase 1 (MTHFD1), methionine synthase reductase (MTRR), methionine adenosyltransferase 1A (MAT1A), methionine adenosyltransferase 2B (MAT2B), folate receptor 1 (FOLR1), cystathionine--synthase (CBS), glutaminase (GLS), DNA methyltransferases 3B (DNMT3B), uracil N-glycosylase 2 (UNG2), adenosine deaminase $(A D A)$ and cysteine dioxygenase (CDO1), were selected for genotyping analysis. Genomic DNA was extracted from $200 \mu$ l of peripheral blood using the QIAamp DNA Blood Mini Kit (QIAGEN, Germany) following the manufacturer's instructions. Purified DNA was evaluated and quantified by agarose gel electrophoresis and spectrophotometer methods. Experimental design and SNP genotyping were carried out by CapitalBio Corporation (Beijing, China) using the Sequenom MassARRAY platform following the manufacturer's instructions. The call rate for each SNP among all the samples was $>95 \%$. In addition, five percent of samples were randomly selected and then repeatedly genotyped with a concordant rate of $100 \%$.

\section{Statistical analysis}

Chi-square tests were carried out to examine Hardy-Weinberg equilibrium (HWE) in the control group. Linkage disequilibrium between SNPs was calculated as $D^{\prime}$ and $R^{2}$ values. The SNPs with strong linkage disequilibrium will be constructed as haplotypes for further analysis.

Unconditional logistic regression methods were used to assess: (1) associations between SNPs polymorphisms and BC risk (statistical significance should be after Benjamini \& Yekutieli correction for multiple testing, $p<0.05$ ). (2) associations between adherence to the MDP and BC risk. (3) associations between adherence to the MDP and BC risk stratified by SNPs genotype.

The potential confounders included are age at diagnosis for cases or enrollment for controls (by years), area (urban, rural), education (ordered as illiterate and primary, middle and high school, university and above), tobacco smoking (no, or yes: including smoking and second-hand smoking 3day/week), moderate physical activity (minutes/day), oral contraceptives use (no, or yes: current use or ever use), hormone replacement therapy (no, or yes: current use or ever use), family history of breast cancer (no, or yes: in a first-degree relative), history of benign breast disease (no, or yes: including lactation mastitis, plasma cell mastitis, cyclomastopathy, fibroadenoma of breast, galactocele), age at menarche (by years), parity $(0,1,2,3)$, age at first full-term delivery (by years), breastfeeding (no, or yes), body mass index (BMl; in $\mathrm{kg} / \mathrm{m}^{2}$ ).

A priori causal model of the causal relationship between the one-carbon metabolism pathway, Mediterranean dietary pattern (MDP) and breast cancer was constructed to determine which confounders need to be adjusted, presenting as a directed acyclic graph (DAG)(Greenland, et al. 1999) (Supplemental Figure 1).

The test of interaction between each SNP genotype and adherence to the MDP were based on both multiplicative model and addictive model. Multiplicative model based on likelihood ratio test and addictive model based on Bootstrap estimation. 
All subjects were included in the present analysis, with the missing covariates data imputed by using the fully conditional specification Multivariate Imputation by Chained Equations method(Azur, et al. 2011). All analyses were performed with R version 4.0.0 (The R Project for Statistical Computing, USA; http://www.rproject. org/).

\section{Results}

From Nov 2013 to Nov 2014, a total of 1410 newly diagnosed breast cancer cases were identified in Wuxi City, 1072 cases meeting the inclusion criteria and 818 of them were recruited in this study. 1072 controls were screened and 935 of them were recruited. Of the 818 cases and 935 controls, the demographic characteristics and anthropometric measures of the subjects stratified by menopausal status are presented in Supplemental Table 1. The priori causal model (using the DAG) that reflects the current knowledge about the relationship between the one-carbon metabolism pathway, Mediterranean dietary pattern (MDP) and breast cancer is shown in Supplemental Figure 1. The minimal sufficient adjustment sets included age, area, education, tobacco smoking, moderate physical activity, oral contraceptives use, hormone replacement therapy, family history of breast cancer, history of benign breast disease, age at menarche, parity, age at first full-term delivery, breastfeeding and body mass index.

\section{Characteristics and frequencies of one-carbon metabolism genes}

Genotype and allele frequencies of the 15 SNPs in eleven one-carbon metabolism pathway-related genes (MTHFD1, MTRR, MAT1A, MAT2B, CDO1, FOLR1, $C B S, G L S, D N M T 3 B, U N G 2, A D A$ ) among cases and controls are shown in Table 1. Because there is no genetic variation (minor allele frequency less than 0.05 was found in the MTHFD1 T>C (rs2230491), MAT1A T>C (rs10887718), CBS T>C (rs11701048), GLST>C (rs12185688) and DNMT3B G>A (rs13045669), they will not be introduced in the following analyses for the association of BC risk and the interaction of diet. The genotype frequencies of the SNPs included in the control group did not deviate from Hardy-Weinberg equilibrium (HWE).

\section{Associations between single-nucleotide polymorphisms (SNPs) and breast cancer risk}

In both adjusted and unadjusted model, women homozygous for the variant of the 10 SNPs: MTHFD1 G>A (rs11627387), MTHFD1 T>C (rs2281603), MTHFD1 $\mathrm{G}>\mathrm{A}($ (rs8003567) , MTRR G>C (rs16879334), MTRR T>C (rs2287780), MAT2B C>A (rs4869087), CDO1 G>C (rs34869), FOLR1 T>G (rs10501409), UNG2 G>A (rs231622), ADA G>A囚rs244072खdemonstrated no statistically significant $(p<0.005)$ increased pre- and post-menopausal BC risk, when compared to the wildtype carriers, as shown in Supplemental Table 2.

For linkage disequilibrium analysis, two considerable degree of linkage disequilibrium were observed between the $M T R R$ T $>C$ ( $r s 227780$ ) and $M T R R$ G $>C$ (rs16879334) $\left(\mathrm{D}^{\prime}=0.99, \mathrm{r}^{2}=0.99\right)$ and MTHFD1 G>A (rs8003567) and MTHFD1 G>A (rs11627387) $\left(\mathrm{D}^{\prime}=0.96, \mathrm{r}^{2}=0.35\right)$, as shown in Figure 1. Haplotypes with a frequency greater than 0.03 were constructed in the case group and control group, but no significant difference was found in the distribution of haplotypes between cases and the controls, as shown in Supplemental Table 3.

\section{Associations between adherence to the Mediterranean dietary pattern and breast cancer risk, stratified by the SNPs genotypes}

A higher aMED score indicated a lower postmenopausal BC risk with a $p$-trend value $<0.0000$, the OR value is 0.54 ( $95 \% \mathrm{Cl}: 0.38-0.78$ ) for comparing top with bottom quartiles, and OR value for per 1 point increase of the aMED is 0.80 (95\% $\mathrm{Cl}$ : 0.70-0.90). However, no significant association was found among premenopausal women (Table 2).

Among postmenopausal women, we found that the association between MDP and BC risk was affected by the SNPs genotype. Stratified by the genotypes of a dominant model (e.g., $A A+A B$ versus $B B, A$ is the risk allele) and an additive model (e.g., $A A$ versus $A B$ versus $B B, A$ is the risk allele), with the increasing number of variant allele, the protective effect against BC risk from high adherence to the MDP was gradually weakened and disappeared. While for MTHFD1 (rs8003567) and CDO1 (rs34869) genotypes, women with homozygous were less affected by high adherence to the MDP than to women who with heterozygotes. The results were shown in Table $\mathbf{3}$ and Figure 2, and the alternate Mediterranean Dietary Pattern score calculated as a continuous variable and a quartile variable, separately.

\section{Interaction analyses between high adherence to the Mediterranean dietary pattern, One-carbon metabolism genes and breast cancer risk}

In the interaction analyses, the wild-type genotype of the SNPs at the lowest quartile values of aMED was used as the reference group. We found a nominal statistical significance of the relevant interaction between MTHFD1 G>A (rs8003567) polymorphisms with postmenopausal breast cancer risk based on the additive and dominant genotypic effects ( $P$-interaction=0.0260 for additive genotypic effects and $P$-interaction=0.0465 for dominant genotypic effects, based on multiplication model), Table 3. However, this interaction is no longer significant under the Benjamini \& Yekutieli correction for multiple comparisons. The detail was shown in Supplemental Table 4 and Table 5.

\section{Discussion}

To our knowledge, this is the first study performed in China that investigated the associations between genetic variants in one-carbon metabolism genes and $\mathrm{BC}$ risk as well as their interactions with MDP on BC risk. Among 10 SNPs involved in the present study, none of them were statistically significantly associated with the risk of $\mathrm{BC}$ despite the menopausal status. However, some meaningful results were found when analyzing the associations between high adherence to MDP and BC risk based on the stratification of genotypes of the SNPs studied. The association between high adherence to MDP and risk 
reduction of BC was affected by the SNPs genotype associated with the one-carbon metabolism pathway. Our study suggests that the one carbon-metabolism genes may act as modifiers between diet and BC risk.

The numerous health benefits of the Mediterranean dietary pattern are widely recognized. Since this dietary pattern is composed of many foods rich in fiber and antioxidants, such as flavonoids, vitamins and carotenoids, it has been linked to the prevention of various diseases, including several common cancers. The underlying mechanisms by which the Mediterranean dietary pattern modulated BC risk were previously identified as the decrease of endogenous estrogens,(Carruba, et al. 2006) neutralization of free radicals to prevent DNA damage(Visioli, et al. 2004) as well as reduction of oxidative stress(Mitjavila, et al. 2013). Although an inverse association of adherence Mediterranean dietary pattern with BC risk has been unveiled by many studies(van den Brandt and Schulpen 2017), such observation failed to be confirmed by others,(Couto, et al. 2013, Demetriou, et al. 2012) or was confined to specific subject subgroups defined by menopausal status(Buckland, et al. 2013, Trichopoulou, et al. 2010). The inconsistent results across different studies may be attributed to differences in study design, racial background, sample size and dietary assessment method. Meanwhile, the influences of unknown genetic variations on BC risk as potential effect modifiers cannot be ignored. Our findings demonstrated that compliance with the Mediterranean diet pattern decrease the risk of breast cancer among post- but not pre-menopausal women. Furthermore, SNPs of one carbon-metabolism gene could modulate the association found above.

The nutrients associated with one-carbon metabolism could not naturally be manufactured by the human body, which means they need to be obtained from foods. The disruption on the one-carbon metabolism pathway could interfere with DNA-repair, DNA-replication, and gene expression regulation, which could be highly carcinogenetic(Kim 2004, Locasale 2013). That has motivated lots of studies focusing on the potential link between the nutrients associated with onecarbon metabolism pathway and carcinogenesis(Milne, et al. 2017, Weinstein, et al. 2006). A previous study showed that SNPs of one-carbon metabolism gene have interactions with folate intake to affect the BC risk(Kakkoura, et al. 2017). However, most previous studies focus on the effect of individual nutrients rather than a combination of foods(Lissowska, et al. 2007), which have been limited in interpreting the high degree of intercorrelation among various nutrients. These associations were always weak because it is hard to attribute effects to single independent component foods(Kim 2004). In this context, we choose a 'posterior' dietary pattern instead of individual nutrients, which could better capture specific diet characteristics and cumulative effects of nutrients.

The Mediterranean dietary pattern is characterized by high consumption of vegetables, fruit, legumes, and fish, rich in folate, choline, vitamins and methionine. Sufficient levels of micronutrients play an important role in the one-carbon metabolism(Park, et al. 2012, Woodside, et al. 2005), because specific enzymes and co-enzymes in one-carbon metabolism require ample quantities of dietary micronutrients (e.g., folate, methionine and other specific amino acids and $B_{2}$, $B_{6}$ and $\mathrm{B}_{12}$ and other vitamins), as substrates to achieve their biological functions(Lucock 2000, Stevens, et al. 2007).

One-carbon metabolism is interconnected to the biological processes of DNA methylation and DNA synthesis(Xu and Chen 2009). Both processes are thought to play key roles in carcinogenesis(Lewis, et al. 2006, Maruti, et al. 2009). DNA methylation is an epigenetic mechanism by which cells regulate gene expression, which involves the addition of a methyl (-CH3) to the 5-carbocytosine residue, predominantly in the cytosine guanine dinucleotide (CPGs). Dietary micronutrients involved in one-carbon metabolism play an essential role in DNA methylation, such as folic acid, choline, betaine, riboflavin, vitamins $B_{6}$ and $B_{12}$, and the amino acid methionine (Figure 3)(Crider, et al. 2012, Locasale 2013). Especially folic acid (or called vitamin $B_{9}$ ), whose role is crucial in the DNA methylation process, producing the methyl group donor, S-adenosylmethionine.

A nominally significant interaction result of MTHFD1 G>A (rs8003567) also implies that one-carbon metabolism genes may be related to diet and BC risk by affecting DNA methylation. The MTHFD1 gene product is a multifunctional enzyme possessing the activities of methylene-THF dehydrogenase, methenyl-THF cyclohydrolase and formyl-THF synthetase in one-carbon metabolism pathway(MacFarlane, et al. 2011). It usually catalyzes sequential and reversible reactions in multiple conversion of tetrahydrofolate (THF), the active form of folate, into 5,10 methylene-THF, which is essential for the de novo purine and thymidylate synthesis as well as the supply of one-carbon units for subsequent DNA methylation. The deficiency or dysregulation of the MTHFD1 enzyme may influence cell division and global methylation pattern, eventually contributing to tumorigenesis(Ding, et al. 2018, MacFarlane, et al. 2011). Since rs 8003567 located in the intronic region of the MTHFD1 gene and no disease-related studies on SNPs have been reported before, another possible explanation cannot be excluded that there are additional functional genetic variants in linkage disequilibrium with these two SNPs that modify BC risk in Chinese female population. However, the interpretation of a nominally significant interaction should be cautious, because the corrected $P$-values for multiple comparisons is no longer significant, the gene-diet interaction obtained may be a false positive result. Thus, replication of the findings in other independent studies is needed before the firm conclusions can be drawn.

However, the relationship between nutrients and DNA methylation is complicated, and there is no unified conclusion now. A recent study in rural African women support that one-carbon nutrient may affect methylation levels, dietary intake of one-carbon metabolites and cofactors in diet fluctuates with seasons. The concentration of biomarkers of maternal carbon metabolism nutrients during pregnancy was associated with the methylation of metastable epi-alleles in DNA from birth infant's lymphocytes and hair follicles. Specifically, plasma concentrations of riboflavin and vitamin $B_{6}$ indicate this association(DominguezSalas, et al. 2014), and previous studies(Dominguez-Salas, et al. 2013) had shown a positive correlation between the two biomarkers and carefully measured dietary intake. However, a recent big cross-sectional study included 5186 adults does not found any log-linear association between the intake of one-carbon metabolic nutrients and individual $\mathrm{CpG}$ methylation.

The strength of our study is that we focus on the genetic variants in the one-carbon metabolism pathway associated with BC risk linking a specific dietary pattern. Most of the previous studies historically focused on individual nutrients(Kim, et al. 2016, Matejcic, et al. 2017) could not capture the complicated interrelationships among nutrients and their cumulative effects(Turati, et al. 2018). The dietary pattern is a combination of food groups rather than isolated nutrients. A 'posterior' Mediterranean dietary pattern contains adequate levels of micronutrients associated with one-carbon metabolisms, such as Vitamins $B_{2}, B_{6}, B_{12}$, folate and choline, which are all involved in DNA methylation and synthesis(Mas, et al. 2007). Thus, it may be more predictive for vivo situation(Brennan, et al. 2010, Gerber 2003) and interpretative for disease risk and biological mechanism. 
Several limitations should also be taken into account in our study. First, we did not obtain adequate information on various subtypes of breast cancer, such as hormone receptor status. The BC risk based on the stratification analysis of breast cancer subtypes is not able to be further evaluated. Second, although we examined several SNPs in multiple key genes thought to be important in the one-carbon metabolism pathway, some other potential polymorphic sites may not be involved in the present study. Third, data were collected from a case-control study, which might be partially influenced by the biases inherent in case-control designs, we analyzed the effect of selection bias in Supplemental Figure 3. Finally, since the number of cases and controls enrolled in this project is relatively small, the associations we founded requires replication in other larger sample independent studies. Further work should assess associations of BC risk and the concentrations of these nutrients in plasma associated with one-carbon metabolism and DNA methylation.

\section{Conclusion}

In conclusion, our results support that the SNPs in one-carbon metabolism genes modify the effect of high adherence to Mediterranean dietary pattern against $\mathrm{BC}$ risk in Chinese women. Genetic variants may as potential effect modifiers influence the association between diet and BC risk.

\section{Declarations}

\section{Ethical approval}

This study was approved by the ethical committee of the Jiangsu Center for Disease Control and Prevention (Jiangsu, China).

\section{Informed consent}

Informed consent was obtained from all individual participants included in the study.

\section{Consent for publication}

Not applicable

\section{Availability of data and materials}

The datasets generated and analyzed during the current study are not publicly available due to the racial genetic characteristics but are available from the corresponding author on reasonable request.

\section{Competing interests}

The authors declare that they have no competing interests

\section{Funding}

This study was supported by World Cancer Research Fund (2011/RFA/473) and Wuxi Young Medical Talents (QNRC035)

\section{Author's Contributions}

All authors contributed to the preparation of the manuscript. PMW and MW (guarantors) had the original idea for the study and carried out the study design, formed the investigator group that obtained the funding, as well as oversaw the study implementation and data collection. JYZ, WL, YQD and YQ assisted data collection and interpreted the data. SC and ZZ carried out the analysis reported in this paper, under the supervision of PMW and MW, and also prepared the initial draft of the manuscript. All authors read and approved the final manuscript.

\section{Acknowledgments}

We are grateful to all study participants for their contributions. We thank the entire data collection team. Incident breast cancer cases and controls for this study were collected by Wuxi Center for Disease Control, Jiangsu Center for Disease Control.

\section{References}

1. Azur MJ, Stuart EA, Frangakis C, Leaf PJ (2011) Multiple imputation by chained equations: what is it and how does it work? Int J Methods Psychiatr Res 20: 40-49 doi: 10.1002/mpr.329

2. Bethea TN et al (2015) A case-control analysis of oral contraceptive use and breast cancer subtypes in the African American Breast Cancer Epidemiology and Risk Consortium. 17: 22 doi: 10.1186/s13058-015-0535-x

3. Brennan SF, Cantwell MM, Cardwell CR, Velentzis LS, Woodside JV (2010) Dietary patterns and breast cancer risk: a systematic review and meta-analysis. Am J Clin Nutr 91: 1294-1302 doi: 10.3945/ajcn.2009.28796

4. Brewer HR, Jones ME, Schoemaker MJ, Ashworth A, Swerdlow AJ (2017) Family history and risk of breast cancer: an analysis accounting for family structure. 165: 193-200 doi: 10.1007/s10549-017-4325-2

5. Buckland G et al (2013) Adherence to the mediterranean diet and risk of breast cancer in the European prospective investigation into cancer and nutrition cohort study. 132: 2918-2927 doi: 10.1002/ijc.27958 
6. Carruba $\mathrm{G}$ et al (2006) A traditional Mediterranean diet decreases endogenous estrogens in healthy postmenopausal women. 56: 253-259 doi: $10.1207 /$ s15327914nc5602_18

7. Couto E, Sandin S, Lof M, Ursin G, Adami HO, Weiderpass E (2013) Mediterranean dietary pattern and risk of breast cancer. 8: e55374 doi: 10.1371/journal.pone.0055374

8. Crider KS, Yang TP, Berry RJ, Bailey LB (2012) Folate and DNA methylation: a review of molecular mechanisms and the evidence for folate's role. Adv Nutr 3: 21-38 doi: 10.3945/an.111.000992

9. Demetriou CA et al (2012) The mediterranean dietary pattern and breast cancer risk in Greek-Cypriot women: a case-control study. 12 : 113 doi: $10.1186 / 1471-2407-12-113$

10. Deschasaux $\mathrm{M}$ et al (2017) Are self-reported unhealthy food choices associated with an increased risk of breast cancer? Prospective cohort study using the British Food Standards Agency nutrient profiling system. 7: e13718 doi: 10.1136/bmjopen-2016-013718

11. Ding K, Jiang J, Chen L, Xu X (2018) Methylenetetrahydrofolate Dehydrogenase 1 Silencing Expedites the Apoptosis of Non-Small Cell Lung Cancer Cells via Modulating DNA Methylation. 24: 7499-7507 doi: 10.12659/MSM.910265

12. Dominguez-Salas $P$ et al (2013) DNA methylation potential: dietary intake and blood concentrations of one-carbon metabolites and cofactors in rural African women. Am J Clin Nutr 97: 1217-1227 doi: 10.3945/ajcn.112.048462

13. Dominguez-Salas P et al (2014) Maternal nutrition at conception modulates DNA methylation of human metastable epialleles. Nat Commun 5: 3746 doi: 10.1038/ncomms4746

14. Ellingjord-Dale M, Vos L, Tretli S, Hofvind S, Dos-Santos-Silva I, Ursin G (2017) Parity, hormones and breast cancer subtypes - results from a large nested case-control study in a national screening program. 19: 10 doi: 10.1186/s13058-016-0798-x

15. Fung TT et al (2005) Diet-quality scores and plasma concentrations of markers of inflammation and endothelial dysfunction. 82: 163-173 doi: 10.1093/ajcn.82.1.163

16. Gerber M (2003) Biofactors in the Mediterranean diet. Clin Chem Lab Med 41: 999-1004 doi: 10.1515/CCLM.2003.153

17. Greenland S, Pearl J, Robins JM (1999) Causal diagrams for epidemiologic research. Epidemiology 10: 37-48 doi:

18. Kakkoura MG et al (2017) Mediterranean diet-gene interactions: A targeted metabolomics study in Greek-Cypriot women. Mol Nutr Food Res $6110.1002 / \mathrm{mnfr} .201600558$

19. Kim SJ et al (2016) Plasma folate, vitamin B-6, and vitamin B-12 and breast cancer risk in BRCA1- and BRCA2-mutation carriers: a prospective study. 104: 671-677 doi: 10.3945/ajcn.116.133470

20. Kim YI (2004) Folate and DNA methylation: a mechanistic link between folate deficiency and colorectal cancer? Cancer Epidemiol Biomarkers Prev 13: 511-519 doi:

21. Lewis SJ, Harbord RM, Harris R, Smith GD (2006) Meta-analyses of observational and genetic association studies of folate intakes or levels and breast cancer risk. J Natl Cancer Inst 98: 1607-1622 doi: 10.1093/jnci/djj440

22. Lissowska J et al (2007) Genetic polymorphisms in the one-carbon metabolism pathway and breast cancer risk: a population-based case-control study and meta-analyses. Int J Cancer 120: 2696-2703 doi: 10.1002/ijc.22604

23. Locasale JW (2013) Serine, glycine and one-carbon units: cancer metabolism in full circle. Nat Rev Cancer 13: 572-583 doi: 10.1038/nrc3557

24. Lucock M (2000) Folic acid: nutritional biochemistry, molecular biology, and role in disease processes. Mol Genet Metab 71: 121-138 doi: $10.1006 /$ mgme.2000.3027

25. Ma E et al (2009) Dietary intake of folate, vitamin B6, and vitamin B12, genetic polymorphism of related enzymes, and risk of breast cancer: a casecontrol study in Brazilian women. 9: 122 doi: 10.1186/1471-2407-9-122

26. MacFarlane AJ, Perry CA, McEntee MF, Lin DM, Stover PJ (2011) Mthfd1 is a modifier of chemically induced intestinal carcinogenesis. 32: 427-433 doi: 10.1093/carcin/bgq270

27. Maruti SS, Ulrich CM, Jupe ER, White E (2009) MTHFR C677T and postmenopausal breast cancer risk by intakes of one-carbon metabolism nutrients: a nested case-control study. Breast Cancer Res 11: R91 doi: 10.1186/bcr2462

28. Mas $\mathrm{S}$ et al (2007) Lower specific micronutrient intake in colorectal cancer patients with tumors presenting promoter hypermethylation in p16(INK4a), p4(ARF) and hMLH1. Anticancer Res 27: 1151-1156 doi:

29. Matejcic $\mathrm{M}$ et al (2017) Biomarkers of folate and vitamin B12 and breast cancer risk: report from the EPIC cohort. 140: 1246-1259 doi: $10.1002 /$ ijc.30536

30. Milne RL et al (2017) Cohort Profile: The Melbourne Collaborative Cohort Study (Health 2020). Int J Epidemiol 46: 1757 doi: $10.1093 /$ ije/dyx085

31. Miranti EH et al (2017) Low vitamin B12 increases risk of gastric cancer: A prospective study of one-carbon metabolism nutrients and risk of upper gastrointestinal tract cancer. 141: 1120-1129 doi: 10.1002/ijc.30809

32. Mitjavila MT et al (2013) The Mediterranean diet improves the systemic lipid and DNA oxidative damage in metabolic syndrome individuals. A randomized, controlled, trial. 32: 172-178 doi: 10.1016/j.clnu.2012.08.002

33. Neuhouser ML et al (2015) Overweight, Obesity, and Postmenopausal Invasive Breast Cancer Risk: A Secondary Analysis of the Women's Health Initiative Randomized Clinical Trials. 1: 611-621 doi: 10.1001/jamaoncol.2015.1546

34. Park JY et al (2012) Comparison of standardised dietary folate intake across ten countries participating in the European Prospective Investigation into Cancer and Nutrition. Br J Nutr 108: 552-569 doi: 10.1017/S0007114511005733

35. Platek ME et al (2009) Alcohol consumption and genetic variation in methylenetetrahydrofolate reductase and 5-methyltetrahydrofolate-homocysteine methyltransferase in relation to breast cancer risk. 18: 2453-2459 doi: 10.1158/1055-9965.EPI-09-0159

Page $7 / 13$ 
36. Shao HB et al (2018) Human methionine synthase A2756G polymorphism increases susceptibility to prostate cancer. 10: 1776-1788 doi: 10.18632/aging.101509

37. Soltani S, Jayedi A, Shab-Bidar S, Becerra-Tomas N, Salas-Salvado J (2019) Adherence to the Mediterranean Diet in Relation to All-Cause Mortality: A Systematic Review and Dose-Response Meta-Analysis of Prospective Cohort Studies. Adv Nutr 10: 1029-1039 doi: 10.1093/advances/nmz041

38. Stevens VL, McCullough ML, Pavluck AL, Talbot JT, Feigelson HS, Thun MJ, Calle EE (2007) Association of polymorphisms in one-carbon metabolism genes and postmenopausal breast cancer incidence. Cancer Epidemiol Biomarkers Prev 16: 1140-1147 doi: 10.1158/1055-9965.EPI-06-1037

39. Torres D et al (2017) Prevalence and Penetrance of BRCA1 and BRCA2 Germline Mutations in Colombian Breast Cancer Patients. 7: 4713 doi: 10.1038/s41598-017-05056-y

40. Trichopoulou A et al (2014) Definitions and potential health benefits of the Mediterranean diet: views from experts around the world. 12: 112 doi: $10.1186 / 1741-7015-12-112$

41. Trichopoulou A, Bamia C, Lagiou P, Trichopoulos D (2010) Conformity to traditional Mediterranean diet and breast cancer risk in the Greek EPIC (European Prospective Investigation into Cancer and Nutrition) cohort. 92: 620-625 doi: 10.3945/ajcn.2010.29619

42. Trinh T et al (2015) Background risk of breast cancer and the association between physical activity and mammographic density. 17: 50 doi: 10.1186/s13058-015-0565-4

43. Turati F et al (2018) Mediterranean Diet and Breast Cancer Risk. 1010.3390/nu10030326

44. van den Brandt PA, Schulpen M (2017) Mediterranean diet adherence and risk of postmenopausal breast cancer: results of a cohort study and metaanalysis. Int J Cancer 140: 2220-2231 doi: 10.1002/ijc.30654

45. Visioli F, Grande S, Bogani P, Galli C (2004) The role of antioxidants in the mediterranean diets: focus on cancer. 13: $337-343$ doi:

46. Weinstein SJ, Stolzenberg-Solomon R, Pietinen P, Taylor PR, Virtamo J, Albanes D (2006) Dietary factors of one-carbon metabolism and prostate cancer risk. Am J Clin Nutr 84: 929-935 doi: 10.1093/ajcn/84.4.929

47. Woodside JV, McCall D, McGartland C, Young IS (2005) Micronutrients: dietary intake v. supplement use. Proc Nutr Soc 64: 543-553 doi: $10.1079 /$ pns2005464

48. Wu JL et al (2016) MTHFR c.677C>T Inhibits Cell Proliferation and Decreases Prostate Cancer Susceptibility in the Han Chinese Population in Shanghai. 6: 36290 doi: $10.1038 /$ srep36290

49. Xu X, Chen J (2009) One-carbon metabolism and breast cancer: an epidemiological perspective. J Genet Genomics 36: 203-214 doi: 10.1016/S16738527(08)60108-3

50. Zhao W, Hasegawa K, Chen J (2002) The use of food-frequency questionnaires for various purposes in China. Public Health Nutr 5: 829-833 doi: $10.1079 /$ phn2002374

\section{Tables}

Table 1.

Genotype and minor allele frequencies for the single-nucleotide polymorphisms (SNPs) in one-carbon metabolism pathway related genes

\begin{tabular}{|c|c|c|c|c|c|c|c|}
\hline \multirow[t]{2}{*}{ Gene } & \multirow[t]{2}{*}{ dbSNP IDa } & \multirow{2}{*}{$\begin{array}{l}\text { Risk(variant) } \\
\text { allele }\end{array}$} & \multicolumn{3}{|c|}{ Cases/Controls ${ }^{b}$} & \multirow{2}{*}{$\begin{array}{l}\mathrm{MAF}^{\mathrm{C}} \\
\text { } \text { Cases/Controls } \rrbracket\end{array}$} & \multirow{2}{*}{$\begin{array}{l}\text { Hardy-Weinberg } \\
(P \text {-value })\end{array}$} \\
\hline & & & Wild-type & Heterozygote & Homozygote & & \\
\hline \multirow[t]{4}{*}{ MTHFD1 } & rs11627387 & $A$ & $276 / 334$ & $390 / 424$ & $128 / 160$ & $0.41 / 0.41$ & 0.45 \\
\hline & rs2230491 & C & $801 / 922$ & $0 / 2$ & $0 / 0$ & $0 / 0$ & - \\
\hline & rs2281603 & C & $297 / 360$ & $393 / 438$ & $104 / 119$ & $0.38 / 0.37$ & 0.73 \\
\hline & rs8003567 & $A$ & $503 / 585$ & $263 / 291$ & $33 / 46$ & $0.20 / 0.21$ & 0.46 \\
\hline \multirow[t]{2}{*}{ MTRR } & rs16879334 & C & $551 / 633$ & $229 / 267$ & $16 / 19$ & $0.16 / 0.17$ & 0.32 \\
\hline & rs2287780 & C & $552 / 635$ & $231 / 269$ & $16 / 20$ & $0.16 / 0.17$ & 0.39 \\
\hline MAT1A & rs10887718 & $\mathrm{C}$ & $767 / 890$ & $33 / 35$ & $0 / 0$ & $0.02 / 0.02$ & - \\
\hline MAT2B & rs4869087 & A & $607 / 718$ & $177 / 189$ & $15 / 15$ & $0.13 / 0.12$ & 0.82 \\
\hline CD01 & rs34869 & $\mathrm{C}$ & $356 / 402$ & $342 / 427$ & $100 / 99$ & $0.34 / 0.34$ & 0.66 \\
\hline FOLR1 & rs10501409 & G & $414 / 496$ & $325 / 376$ & $59 / 59$ & $0.28 / 0.27$ & 0.48 \\
\hline CBS & rs11701048 & C & $782 / 907$ & $17 / 15$ & $0 / 0$ & $0.01 / 0.01$ & - \\
\hline GLS & rs12185688 & C & $800 / 924$ & $800 / 924$ & $0 / 0$ & $0 / 0$ & - \\
\hline DNMT3B & rs13045669 & $A$ & $800 / 922$ & $800 / 922$ & $0 / 0$ & $0 / 0$ & - \\
\hline UNG2 & rs231622 & A & $264 / 299$ & $388 / 450$ & $141 / 171$ & $0.43 / 0.43$ & 0.98 \\
\hline ADA & rs244072 & $A$ & $542 / 641$ & $231 / 252$ & $24 / 26$ & $0.18 / 0.17$ & 0.9 \\
\hline
\end{tabular}

Page 8/13 
a SNP identifier based on NCBI dbSNP

${ }^{\mathrm{b}}$ The number of cases and controls may differ from those of study-subjects due to missing genotype values

${ }^{\mathrm{c}} \mathrm{MAF}$ minor allele frequency

d $P$ value from Chi-square test performed for Hardy-Weinberg equilibrium (HWE) evaluation, using only controls

Table 2.

Multivariate-adjusted odd risks (ORs) of breast cancer risk in relation to the alternate Mediterranean Diet Score (aMED), stratified by menopausal status

\begin{tabular}{|lllll|}
\hline aMed & \multicolumn{2}{l|}{ Premenopausal women $(\mathrm{n}=600)$} & \multicolumn{2}{l|}{ Postmenopausal women $(\mathrm{n}=1153)$} \\
\cline { 2 - 5 } & Case/Control & Adjusted OR $(95 \% \mathrm{Cl})$ & Case/Control & Adjusted OR ${ }^{\mathrm{a}, \mathrm{b}}(95 \% \mathrm{Cl})$ \\
\hline Quartile 1 & $52 / 106$ & 1.00 & $190 / 146$ & 1.00 \\
\hline Quartile 2 & $63 / 95$ & $1.34(0.83,2.20)$ & $170 / 133$ & $0.97(0.69,1.35)$ \\
\hline Quartile 3 & $58 / 83$ & $1.55(0.94,2.57)$ & $135 / 128$ & $0.77(0.54,1.09)$ \\
\hline Quartile 4 & $41 / 102$ & $0.90(0.53,1.53)$ & $109 / 142$ & $0.54(0.38,0.78)$ \\
\hline P-trend & & 0.8241 & & 0.0005 \\
\hline Per SD increase & & $0.98(0.82,1.17)$ & & $0.80(0.70,0.90)$ \\
\hline
\end{tabular}

a Adjusted for age at diagnosis for cases or enrollment for controls, area, education, tobacco smoking, moderate physical activity, oral contraceptives use, hormone replacement therapy, family history of breast cancer, history of benign breast disease, age at menarche, number of full term births, age at first full term delivery, breastfeeding and body mass index.

${ }^{b}$ Adjusted for the menopausal age.

\section{Table 3.}

Associations between breast cancer risk and adherence to Mediterranean dietary pattern (MDP), stratified by genotypes 


\begin{tabular}{|c|c|c|c|c|c|c|c|c|c|c|c|}
\hline Gene & SNP & Alleles & Cases/Controls ${ }^{a}$ & $\begin{array}{l}\text { Adjusted } \\
\text { OR }(95 \\
\% \mathrm{Cl})^{\mathrm{b}, \mathrm{c}}\end{array}$ & $\begin{array}{l}P \text { - } \\
\text { interaction }^{d}\end{array}$ & Gene & SNP & Alleles & Cases/Controls ${ }^{a}$ & $\begin{array}{l}\text { Adjusted } \\
\text { OR }(95 \\
\% \mathrm{Cl})^{\mathrm{b}, \mathrm{c}}\end{array}$ & $\begin{array}{l}P \\
\text { in }\end{array}$ \\
\hline \multirow[t]{15}{*}{ MTHFD1 } & rs11627387 & GG & $202 / 201$ & $\begin{array}{l}0.80 \\
(0.68 \\
0.94)\end{array}$ & 0.7837 & MAT2B & rs4869087 & $\mathrm{CC}$ & $446 / 417$ & $\begin{array}{l}0.85 \\
(0.76 \\
0.94)\end{array}$ & 0 \\
\hline & & GA & $288 / 239$ & $\begin{array}{l}0.85 \\
(0.74 \\
0.98)\end{array}$ & & & & CA & $129 / 119$ & $\begin{array}{l}0.90 \\
(0.74, \\
1.09)\end{array}$ & \\
\hline & & AA & 93/101 & $\begin{array}{l}0.82 \\
(0.65, \\
1.02)\end{array}$ & & & & AA & $12 / 9$ & $\begin{array}{l}1.09 \\
(0.57 \\
2.09)\end{array}$ & \\
\hline & & GG & $202 / 201$ & $\begin{array}{l}0.80 \\
(0.68 \\
0.94)\end{array}$ & 0.8567 & & & CC & $446 / 417$ & $\begin{array}{l}0.85 \\
(0.76 \\
0.94)\end{array}$ & 0 \\
\hline & & $\mathrm{GA}+\mathrm{AA}$ & $381 / 340$ & $\begin{array}{l}0.86 \\
(0.77 \\
0.96)\end{array}$ & & & & $\mathrm{CA}+\mathrm{AA}$ & $141 / 128$ & $\begin{array}{l}0.91 \\
(0.75 \\
1.09)\end{array}$ & \\
\hline & rs2281603 & TT & $215 / 212$ & $\begin{array}{l}0.79 \\
(0.68 \\
0.92)\end{array}$ & 0.1345 & CD01 & rs34869 & GG & $261 / 237$ & $\begin{array}{l}0.93 \\
(0.82, \\
1.06)\end{array}$ & 0 \\
\hline & & $\mathrm{TC}$ & $291 / 254$ & $\begin{array}{l}0.87 \\
(0.75 \\
0.99)\end{array}$ & & & & GC & $253 / 263$ & $\begin{array}{l}0.82 \\
(0.71 \\
0.94)\end{array}$ & \\
\hline & & $\mathrm{CC}$ & $77 / 75$ & $\begin{array}{l}1.00 \\
(0.79 \\
1.26)\end{array}$ & & & & CC & $75 / 47$ & $\begin{array}{l}0.77 \\
(0.54 \\
1.09)\end{array}$ & \\
\hline & & $\mathrm{TT}$ & $215 / 212$ & $\begin{array}{l}0.79 \\
(0.68 \\
0.92)\end{array}$ & 0.1685 & & & GG & $261 / 237$ & $\begin{array}{l}0.93 \\
(0.82 \\
1.06)\end{array}$ & 0 \\
\hline & & $\mathrm{TC}+\mathrm{CC}$ & $368 / 329$ & $\begin{array}{l}0.89 \\
(0.80 \\
1.00)\end{array}$ & & & & $\mathrm{GC}+\mathrm{CC}$ & $328 / 310$ & $\begin{array}{l}0.80 \\
(0.71 \\
0.91)^{\prime}\end{array}$ & \\
\hline & rs8003567 & GG & $373 / 348$ & $\begin{array}{l}0.89 \\
(0.80 \\
1.00)\end{array}$ & 0.0260 & FOLR1 & rs10501409 & TT & $301 / 293$ & $\begin{array}{l}0.84 \\
(0.75 \\
0.96)\end{array}$ & 0 \\
\hline & & GA & $190 / 169$ & $\begin{array}{l}0.81 \\
(0.68 \\
0.95)\end{array}$ & & & & TG & $250 / 213$ & $\begin{array}{l}0.88 \\
(0.77 \\
1.01)\end{array}$ & \\
\hline & & AA & $24 / 27$ & $\begin{array}{l}0.60 \\
(0.38 \\
0.95)\end{array}$ & & & & GG & $36 / 37$ & $\begin{array}{l}1.11 \\
(0.62, \\
2.04)\end{array}$ & \\
\hline & & GG & $373 / 348$ & $\begin{array}{l}0.89 \\
(0.80 \\
1.00)\end{array}$ & 0.0465 & & & $\mathrm{TT}$ & $301 / 293$ & $\begin{array}{l}0.84 \\
(0.75 \\
0.96)\end{array}$ & 0 \\
\hline & & $\mathrm{GA}+\mathrm{AA}$ & $214 / 196$ & $\begin{array}{l}0.79 \\
(0.68 \\
0.92)\end{array}$ & & & & $\mathrm{TG}+\mathrm{GG}$ & $286 / 250$ & $\begin{array}{l}0.88 \\
(0.77 \\
1.00)\end{array}$ & \\
\hline \multirow[t]{6}{*}{ MTRR } & rs16879334 & GG & $415 / 376$ & $\begin{array}{l}0.83 \\
(0.75 \\
0.93)\end{array}$ & 0.3800 & UNG2 & rs231622 & GG & $200 / 175$ & $\begin{array}{l}0.91 \\
(0.77 \\
1.06)\end{array}$ & 0 \\
\hline & & $\mathrm{GC}$ & $159 / 152$ & $\begin{array}{l}0.91 \\
(0.77 \\
1.07)\end{array}$ & & & & GA & $272 / 282$ & $\begin{array}{l}0.81 \\
(0.71 \\
0.92)\end{array}$ & \\
\hline & & CC & $11 / 13$ & $\begin{array}{l}0.78 \\
(0.37 \\
1.67)\end{array}$ & & & & $A A$ & $112 / 86$ & $\begin{array}{l}0.83 \\
(0.64, \\
1.06)\end{array}$ & \\
\hline & & GG & $415 / 376$ & $\begin{array}{l}0.83 \\
(0.75 \\
0.93)\end{array}$ & 0.3267 & & & GG & $200 / 175$ & $\begin{array}{l}0.91 \\
(0.77 \\
1.06)\end{array}$ & 0 \\
\hline & & $\mathrm{GC}+\mathrm{CC}$ & $170 / 165$ & $\begin{array}{l}0.90 \\
(0.77 \\
1.06)\end{array}$ & & & & $\mathrm{GA}+\mathrm{AA}$ & $384 / 368$ & $\begin{array}{l}0.82 \\
(0.73 \\
0.91)\end{array}$ & \\
\hline & rs2287780 & TT & $416 / 377$ & $\begin{array}{l}0.83 \\
(0.74 \\
0.92)\end{array}$ & 0.2499 & ADA & rs244072 & GG & $405 / 370$ & $\begin{array}{l}0.85 \\
(0.76 \\
0.95)\end{array}$ & 0 \\
\hline
\end{tabular}




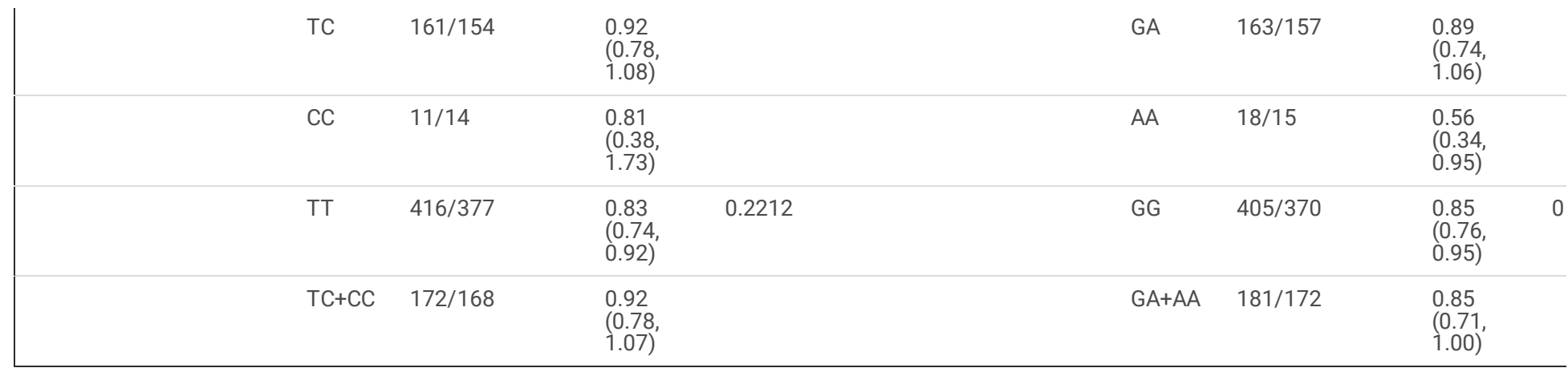

${ }^{a}$ The number of cases and controls may differ from those of study-subjects due to missing genotype values

${ }^{b}$ Adjusted for age at diagnosis for cases or enrollment for controls, area, education, tobacco smoking, moderate physical activity, oral contraceptives use, hormone replacement therapy, family history of breast cancer, history of benign breast disease, age at menarche, number of full term births, age at first full term delivery, breastfeeding and body mass index

${ }^{\mathrm{c}}$ Adjusted for the menopausal age

d P-interaction from Multiplication model result, SNP genotype were as 2 categories or 3categories to calculate the interaction with adherence to MDP on BC risk, respectively.

\section{Figures}

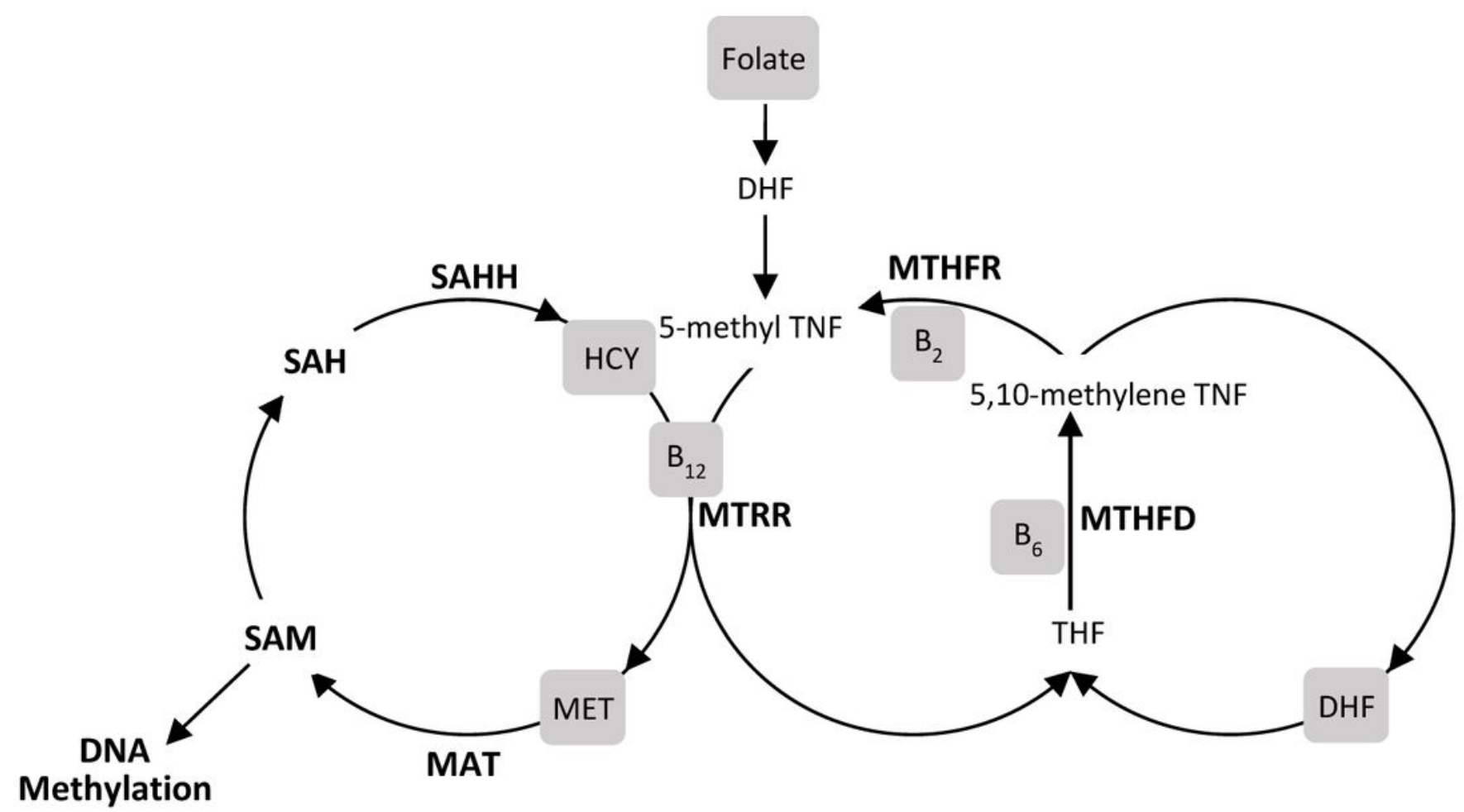

Figure 1

One-carbon metabolism, shading indicates the substrate is obtained via the diet. DHF, dihydrofolate; DMG, dimethyl glycine; SAH, S-adenosylhomocysteine; SAM, S-adenosylmethionine; THF, tetrahydrofolate; DHF, dihydrofolate; MET, methionine; HCY, Homocysteine. 

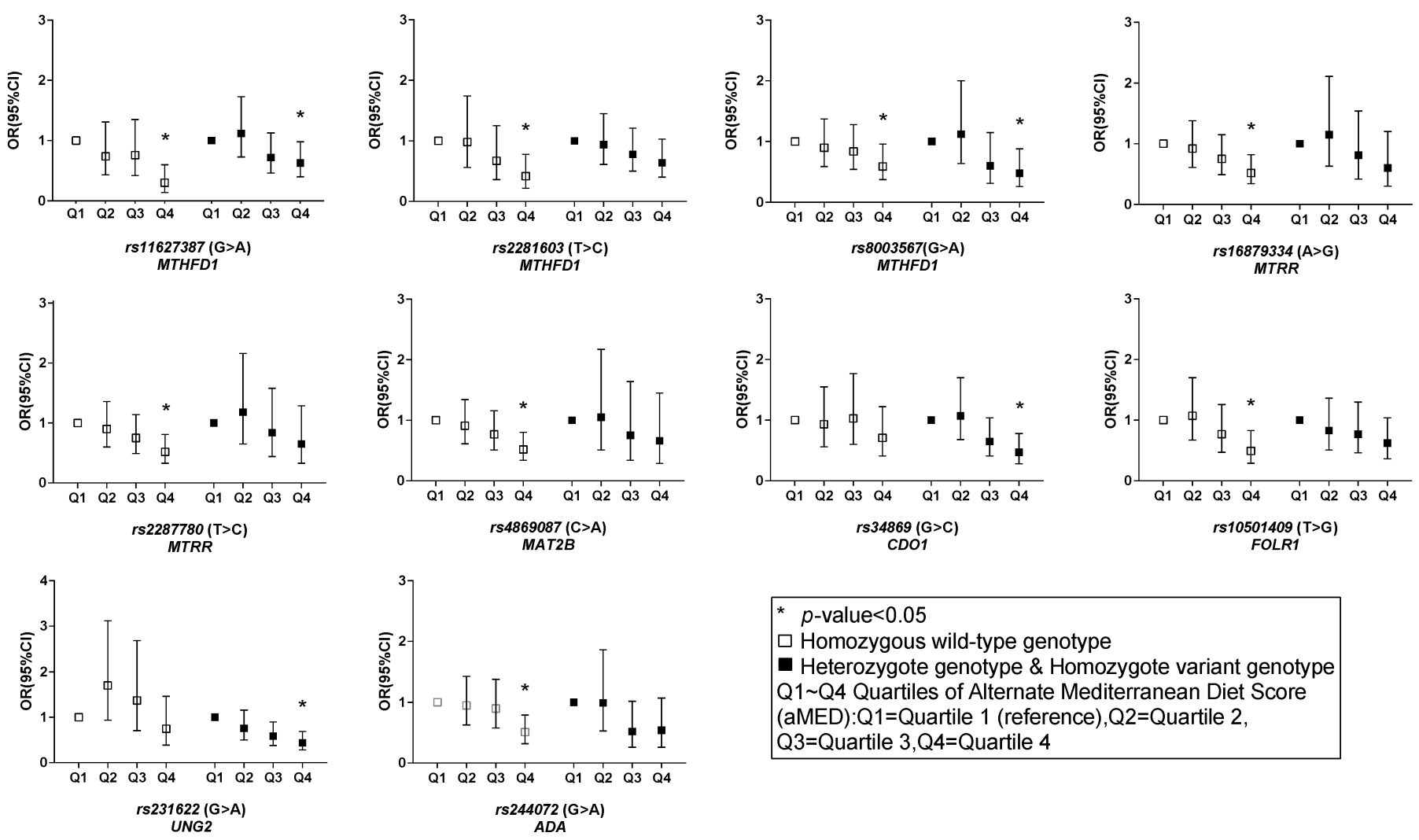

* $p$-value<0.05
$\square$ Homozygous wild-type genotype

Heterozygote genotype \& Homozygote variant genotype Q1 Q4 Quartiles of Alternate Mediterranean Diet Score (aMED):Q1=Quartile 1 (reference),Q2=Quartile 2, Q3=Quartile 3,Q4=Quartile 4

\section{Figure 2}




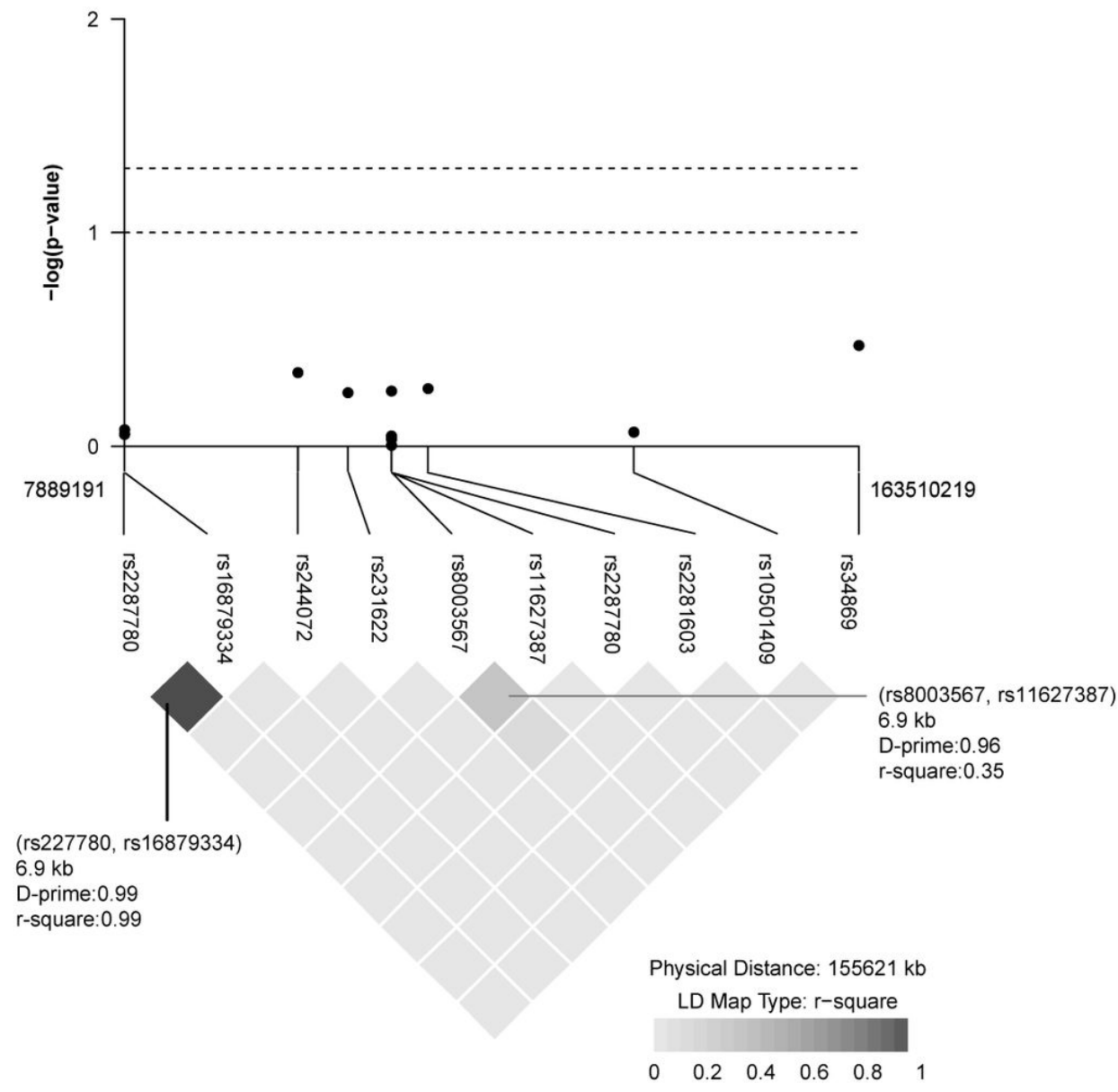

\section{Figure 3}

Logarithm-transformed $\mathrm{P}$ values for the association between breast cancer risk and single-nucleotide polymorphisms (SNPs) of one carbon-metabolism genes and pattern of linkage disequilibrium for tagging SNPs genotyped in carbon-metabolism genes.

\section{Supplementary Files}

This is a list of supplementary files associated with this preprint. Click to download.

- Supplementmateriarevision6.19.docx 